\title{
Geometric frustrated assemblies
}

\section{How geometric frustration shapes twisted fibres, inside and out: competing morphologies of chiral filament assembly}

Authors: Douglas M. Hall and Gregory M. Grason

Interface Focus 7: 20160140 (2017)

\section{Recommended with a Commentary by Roya Zandi, Department of Physics and Astronomy, University of CA, Riverside}

Finding the ground state of geometrically frustrated structures is a long-standing area of research including the canonical problem of Thompson's century old problem. Even the simple problem of organization of spherical particles confined on the surface of a sphere is not yet completely resolved [1]. Recently, some new ways of understanding metric properties of assemblies of 1D line-like elements have shown that this class of problems now extends to a new range of structures. For example, Hall and Grason studied the structure of biological bundles considering the complexity arising from the chirality of filaments and the geometric frustration associated with twisting of the bundle [2]. The origin of this frustration is due to geometrically non-linear constraints that link filament orientation to their spacing [3]. Twisting of the bundle, which involves the collective winding of filaments around the central axis of the bundle, can be driven by molecular chirality that favors local twisting of the neighboring filaments, or instead by some "global mechanical forces" on the system.

Geometric frustration in a twisted bundle plays an important role in its morphology. Since it is impossible to regularly place the strands in a bundle cross section, the system of filament twisting becomes frustrated, which is analogous to the problem of construction of a triangular lattice on a sphere. The previous work by Gompper and Schneider shows that for small enough line tension, crystal domains on a sphere are not stable and form narrow strips [4]. In the paper published in Interfocus, Hall and Grason address a similar problem: how a cohesive chiral bundle respond to the geometric frustration? Or more specifically which of mechanisms do the twisted bundles employ to relax geometric frustration: defects in the interior or reshaping the boundary?

To explore how the ground state free energy or the optimal morphology of bundles depends on the twist and cohesive interaction between them, Hall and Grason consider the following free energy for a chiral bundle,

$$
F=F_{\text {assem }}+F_{\text {elast }}+F_{\text {chiral }}
$$

where $F_{\text {assem }}$ includes the attractive interaction and also surface energy of the bundle resulting from fewer contacts between the filaments at boundaries. The $F_{\text {elast }}$ term corresponds to 


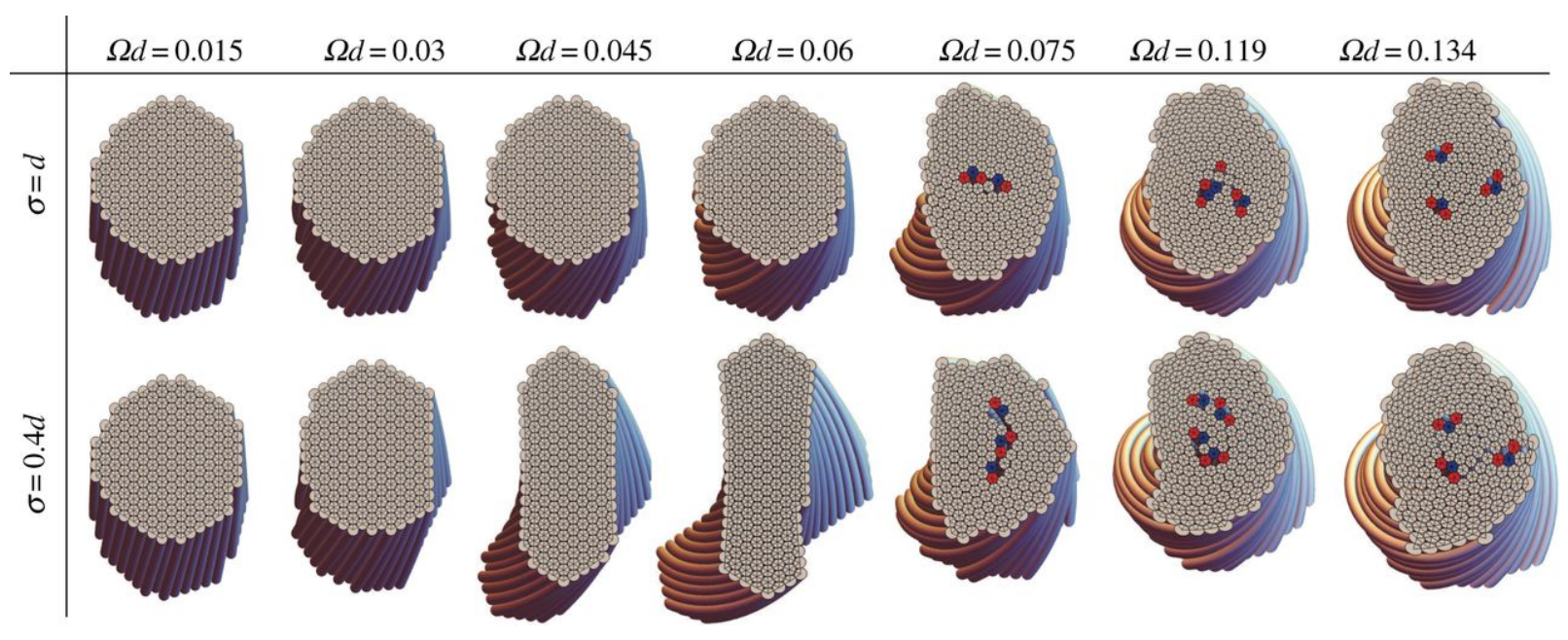

Figure 1: The optimal configuration of 181 filaments obtained in MC simulations, for different values of twist $\Omega d$ with $d$ the diameter of each filament and $\Omega$ the rate of twist. The quantity $\sigma$ indicates the range of attractive interaction. On the top row, $\sigma=d$ corresponds to a relatively ductile material. There is a transition from cylindrical defect-free bundles to defective ones with excess fivefold disclinations. Red (blue) filaments indicate fivefold (sevenfold) disclinations. The bottom row illustrates that for a relatively brittle material, there is an intermediate regime between defect-free and defective cylindrical morphologies, in which anistropic "tape-like" bundles form.

the bending and stretching of filaments and $F_{\text {chiral }}$ is associated with the elastic deformation of a chiral filament due to twisting in a bundle.

Using the above free energy, they calculate the elastic cost of insertion of a disclination. They find under what conditions a bundle keeps its cylindrical shape at the cost of possessing topological defects in the interior instead of forming a defect-free bundle with anisotropic shapes (tapes) and larger surface tension. They show that if the surface tension is high, the filaments prefer to keep their cylindrical shape and a disclination form inside the bundle. However, if the surface energy is low, the cross-sectional shape of the bundle changes and assume a tape-like structure.

Hall and Grason also perform a series of Monte Carlo simulations using a minimal model of cohesive interactions (Lennard Jones potential) between filaments. The results of their Monte Carlo simulations are presented in Fig. 1 in the form of a "shape" diagram versus twisting strength and ductility of filaments. The quantity $\Omega d=\frac{2 \pi d}{P}$ in the figure is the dimensionless twisting rate with the pitch $P$ and strand diameter $d$. The ductility of materials depends on the range of Lennard Jones potential between filaments $\sigma$ compared to the diameter of the filaments. Their result show that the optimal morphology of bundles involving the boundary shape and bulk defects depends on the filament number $N$, the bundle twist and the ductility of inter-filament bonds.

As illustrated in Fig. 1, for ductile bundles $(\sigma / d \sim 1)$, with increasing twist $(\Omega d)$, there is a sharp transition from circular cross section with no disclination to bundles possessing 
topological defects in their interior. The cylindrical shape of the bundles will be preserved after the transition while the topological charge increases with the twist angle. Their results indicate that disclination screens the compression resulting from twisting in a bundle or more specifically, the interaction between twist and positive disclinations is negative.

However, they show that for brittle bundles (small $\sigma / d$ ), there is an intermediate state between defect-free and the defective cylindrical phases. The intermediate phase corresponds to defect-free anisotropic (tape-like) bundles. Quite interestingly, they were able to estimate the stability window of anisotropic tapes in simulations using continuum theory in Eq. 1, which considers the competition between the costs of deforming inter-filament distance through including singular topological defects and the cost of formation of larger anisotropic boundary.

The competition in terms of "defect" vs. "boundary" modes for structural relaxation discussed in the paper of Hall and Grason goes beyond the system of twisted bundles and can elucidate the broader puzzle for similar problems in geometric frustrated assemblies such as packing of all sorts of objects in curved space. Furthermore, it seems that frustration not only shapes the equilibrium ground states, but also it has a significant impact on the non-

equilibrium pathways of assembly. A problem that has already been discussed in the case of assembly of protein cages and viruses [1] and remains an open question: How does frustration shape assembly pathways and non-equilibrium structures in general?

\section{References}

[1] S. Li, P. Roy, A. Travesset, and R. Zandi, Proc. Natl. Acad. Sci. USA 115, 10971 (2018).

[2] Douglas M. Hall and Gregory M. Grason, Interface Focus 7, 20160140 (2017).

[3] Gregory M. Grason, Rev. Mod. Phys. 87, 401 (2015).

[4] S. Schneider and G. Gompper, EPL, 70, 136 (2005). 